\title{
IDENTIFICAÇÃO ELETRÔNICA PARA AVALIAÇÃO DO COMPORTAMENTO DOS SUÍNOS NA FASE DE GESTAÇÃO
}

\author{
KÉSIA O. DA SILVA ${ }^{1}$, IRENILZA DE A. NÄÄS ${ }^{2}$
}

RESUMO: O desempenho eficiente da produção de suínos depende da gestão do rebanho, bem como da nutrição, do controle sanitário, das instalações e de condições ambientais adequadas. O conceito desse tipo de produção está diretamente relacionado com a redução das perdas seletivas e o controle do processo. Cada segmento da produção é controlado de maneira a alcançar a otimização na totalidade do sistema, ou seja, é necessário aplicar conceitos de manejo dos animais. Sendo assim, o presente trabalho apresenta como objetivo o desenvolvimento de modelo matemático para avaliar as interações do ambiente interno da instalação com a preferência térmica dos animais, detectando relação entre a freqüência de acesso ao bebedouro e as condições do ambiente, temperatura ambiente, temperatura de globo negro e umidade relativa, utilizando como ferramenta a identificação eletrônica. Os resultados obtidos por meio do modelo matemático permitiram concluir com precisão a avaliação da preferência térmica dos suínos em relação às variáveis climáticas na fase de gestação.

PALAVRAS-CHAVE: conforte térmico, dispositivos eletrônicos, etologia.

\section{ELECTRONIC IDENTIFICATION FOR EVALUATION OF SWINE BEHAVIOR IN PREGNANCY STAGE}

\begin{abstract}
The efficiency of swine production performance depends on the herd administration, such as good nutrition, sanitary control, facilities and appropriate environmental conditions. The concept of this production model is directly related with the reduction of selective losses and the process control. Each production segment is controlled to reach the optimization in the system totality, it is necessary to apply animals handling concepts, environmental control implementation, diseases control, nutrition control, information concerning in guaranteeing the animal welfare and individual identification. The present work presents as objective the development of the mathematical model to evaluate interactions among the internal atmosphere of the installation and the thermal animals preference, in the expectation of detecting a relationship among the frequency access to the drinking fountain and the atmosphere conditions - temperature, black globe temperature and relative humidity, using as tool the electronic identification. The results obtained by the mathematical model, allowed to conclude accurately the evaluation of the swine thermal preference correlating with the climatic variables in the pregnancy stage.
\end{abstract}

KEYWORDS: thermal comfort, electronic devices, ethnology.

\section{INTRODUÇÃO}

Face a necessidade de atender às crescentes demandas dos consumidores no mundo, aliada à falta de espaço para a expansão da produção animal, há tendência em levar a cadeia alimentícia a procurar a otimização do espaço e, conseqüentemente, o uso apropriado de sistemas de suporte à vida.

Porém, com maiores densidades no confinamento dos animais, a emissão de efluentes e gases pode forçar a sustentabilidade até seus limites.

\footnotetext{
${ }^{1}$ Eng ${ }^{\mathrm{a}}$ Agrícola, Doutora em Construções Rurais e Ambiência, FEAGRI/UNICAMP, Fone: (OXX19) 3402-9402, Piracicaba - SP, kosilva@uol.com.br

${ }^{2}$ Prof. Titular, Departamento de Construções Rurais, FEAGRI/UNICAMP, irenilza@ agr.unicamp.br

Recebido pelo Conselho Editorial em: 7-6-2004

Aprovado pelo Conselho Editorial em: 11-7-2005
}

Eng. Agríc., Jaboticabal, v.25, n.2, p.322-329, maio/ago. 2005 
O atual aumento da produção animal foi permitido pelo uso de inovações tecnológicas, e a conclusão lógica parece ser que, no futuro, o aumento da produção tanto animal como vegetal poderá ser alcançado da mesma forma.

Desde os anos de 1970, vê-se mudança nas necessidades dos consumidores do mundo inteiro, ao mesmo tempo em que as comunicações têm tornado uma realidade a interação entre as culturas. Isso levou a certa padronização nas demandas e, dessa maneira, conduziu o consumidor à busca de alimentos de qualidade, pois está ciente de suas necessidades (NÄ̈̈S, 2001a).

Com a globalização dos mercados a partir da década de 1990, os países desenvolvidos, em especial a União Européia, desenvolveram estratégias para assegurar mercados a seus produtos agrícolas, das quais a segurança alimentar é parte integrante (BELLAVER, 2001).

No Brasil, como no exterior, a atividade suinícola está se tornando agronegócio de alta competitividade. Portanto, para que o suinocultor possa manter-se no mercado, ou, ainda, aumentar a produção a baixo custo e com boa qualidade, é essencial um constante trabalho de modernização, adaptação e melhoria de todos os setores e áreas da linha de produção mediante diferentes métodos (LEITE et al., 2000). Os métodos até então existentes começam a se mostrar ineficientes para garantir um percentual crescente dessa qualidade, pois a garantia somente pode ser efetiva se houver rastreamento confiável do produto, no caso do animal, desde o seu nascimento até o abate e consumo posterior.

A identificação animal faz parte do sistema de produção e apresenta grande importância, pois possibilita a coleta de informações inerentes ao indivíduo. Com essas informações, é possível trabalhar as variáveis do ambiente e correlacioná-las aos elementos de uma população de forma individual ou coletiva. Uma tecnologia inovadora nesse rastreamento é o da identificação eletrônica, que possui uma série de vantagens quando comparada aos métodos tradicionais. Segundo WADE \& MAYHALL (1994), citado por NÄÄS (2001b), alguns estudos realizados demonstram a viabilidade de implantação de circuitos eletrônicos miniaturizados, na forma de circuitos integrados, conhecidos como transponders ou microchips, que implementam a idéia de identificação eletrônica, levando-se em consideração, principalmente, o conforto e o bem-estar dos animais.

Trabalhos de pesquisa desenvolvidos em todo o mundo sobre o comportamento de animais utilizam-se de alguma tecnologia para dar suporte ao levantamento de dados. KORTHALS et al. (1992) apresentaram um trabalho demonstrando suas experiências com transponders para o monitoramento de respostas bioenergéticas. Em suas pesquisas desenvolvidas no centro americano de pesquisa da carne animal, os autores envolveram-se com o monitoramento intensivo de consumo de alimentos, produção de calor e comportamento dos animais, por meio de equipamentos especiais como identificação individual dos animais, comedouros automáticos com pesagem, calorímetros, registro de imagens e registradores de temperatura estacionários e portáteis baseados em microprocessadores.

Em pesquisas realizadas por HAMRITA et al. (1998), foi investigado o uso de sistema de biotelemetria totalmente automático para a medição da temperatura interna de aves. Apesar de a miniaturização dos implantes eletrônicos, como brincos identificadores ou transponders, serem costumeiramente utilizados em produção animal, o uso de equipamentos de biotelemetria para monitoramento de respostas fisiológicas ao estresse térmico não é comum.

PANDORFI (2002), utilizando leitões em abrigos escamoteadores, concluiu que, das ferramentas tecnológicas estudadas, o sistema que se mostrou mais eficiente, na avaliação do comportamento e bem-estar animal, foi a análise de imagem, permitindo correlacionar o perfil de distribuição dos animais e captar o maior número de informações, uma vez que o uso da identificação eletrônica apresentou erro de detecção da presença dos animais, no ambiente, em torno de $21 \%$. 
Sendo assim, o presente trabalho apresenta como objetivo o desenvolvimento de modelo matemático na expectativa de detectar relação entre a freqüência de acesso ao bebedouro e as condições do ambiente, temperatura, temperatura de globo negro e umidade relativa, utilizando como ferramenta a identificação eletrônica.

\section{MATERIAL E MÉTODOS}

O experimento foi conduzido em granja comercial de produção de suínos (Granja Querência) localizada no município de Salto - SP, situada a $23^{\circ} 12^{\prime} 10^{\prime \prime} \mathrm{S}$ e a $47^{\circ} 17^{\prime} 35^{\prime \prime} \mathrm{W}$ e altitude média de $521 \mathrm{~m}$, no centro da região chamada Zona de Depressão Periférica do Estado de São Paulo, entre o Planalto Atlântico e a escarpa da Serra de Botucatu.

Foram avaliadas as interações do ambiente interno com o comportamento dos animais, relacionando a frequiência de acesso ao comedouro e bebedouro com as condições do ambiente, temperatura ambiente, temperatura de globo negro e umidade relativa. Foi confeccionado um esquema geral de comunicação entre os animais identificados que, por intermédio de um protocolo de comunicação, enviou os dados adquiridos para um microcomputador, que continha um software desenvolvido especialmente para esse projeto, sendo desenvolvido modelo matemático para interpretar esses dados. Os sinais captados pelas antenas foram transferidos via radiofrequiência para o computador, os quais foram manipulados por meio de um software desenvolvido.

Para este experimento, foram utilizadas quatro baias da fase de gestação em gaiola, onde os animais foram identificados por meio de transponders da marca TROVAN com 11,5 mm x 2,2 mm implantados na base da orelha das porcas, considerado como o melhor local para o implante (SILVA et al., 2002). Em seguida, as porcas foram pesadas e retornaram às gaiolas. Um datalogger foi instalado no centro geométrico de cada baia, acoplando-se os sensores de temperatura de bulbo seco, bulbo úmido e globo negro. A comunicação entre o datalogger e o microcomputador foi feita via cabo RS485 para melhor transferência dos dados climáticos. Os sensores foram dispostos no galpão, a $1 \mathrm{~m}$ de altura, para melhor representar o microclima do local. Leitoras fixas (antenas), da marca Trovan, foram instaladas próximas ao bebedouro e comedouro. A antena utilizada possui as dimensões de $40 \mathrm{x}$ $40 \mathrm{~cm}$, e seu raio de alcance é de $40 \mathrm{~cm}$. Dessa forma, por meio de um protocolo de comunicação, pôde-se detectar a freqüência de uso, em função das condições do ambiente.

Para fins de coleta de dados, a instalação dos equipamentos foi feita a $40 \mathrm{~cm}$ acima da canaleta pela qual foi conduzida a água, seguindo um padrão de modo a não interferir nos hábitos dos animais e também para evitar danos aos equipamento. Na Figura 1, representam-se de maneira esquemática os detalhes dessa fase.

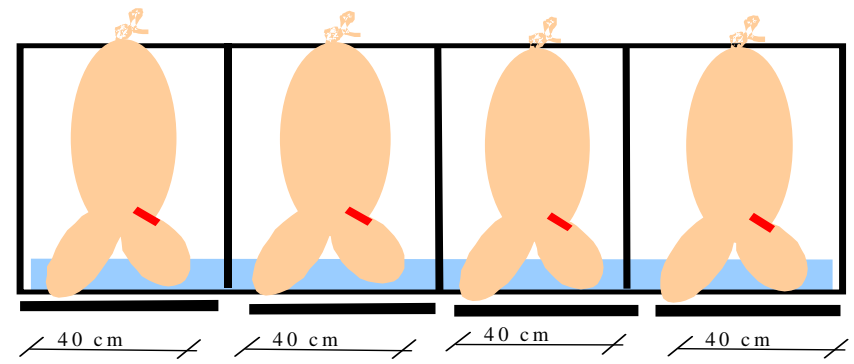

FIGURA 1. Esquema de instalação dos leitores fixos na baia de gestação.

As matrizes foram dispostas lado a lado, de acordo com a sua localização na gaiola. Assim, no momento em que a porca se deslocava para o bebedouro ou comedouro (deve-se ressaltar que nessa fase só é feito um trato por dia, às sete horas da manhã), a leitora fixa efetuava a respectiva leitura do 
sinal do transponder. As grades frontais das gaiolas foram substituídas por caixa de madeira para evitar interferência entre o sinal do transponder e a grade de ferro. As baias em gaiola possuem $0,75 \mathrm{~m}$ de largura por 2,00 $\mathrm{m}$ de comprimento e a canaleta do bebedouro possui $0,3 \mathrm{~m}$ de largura. $\mathrm{O}$ esquema geral dessa etapa do experimento pode ser conferido pela Figura 2.

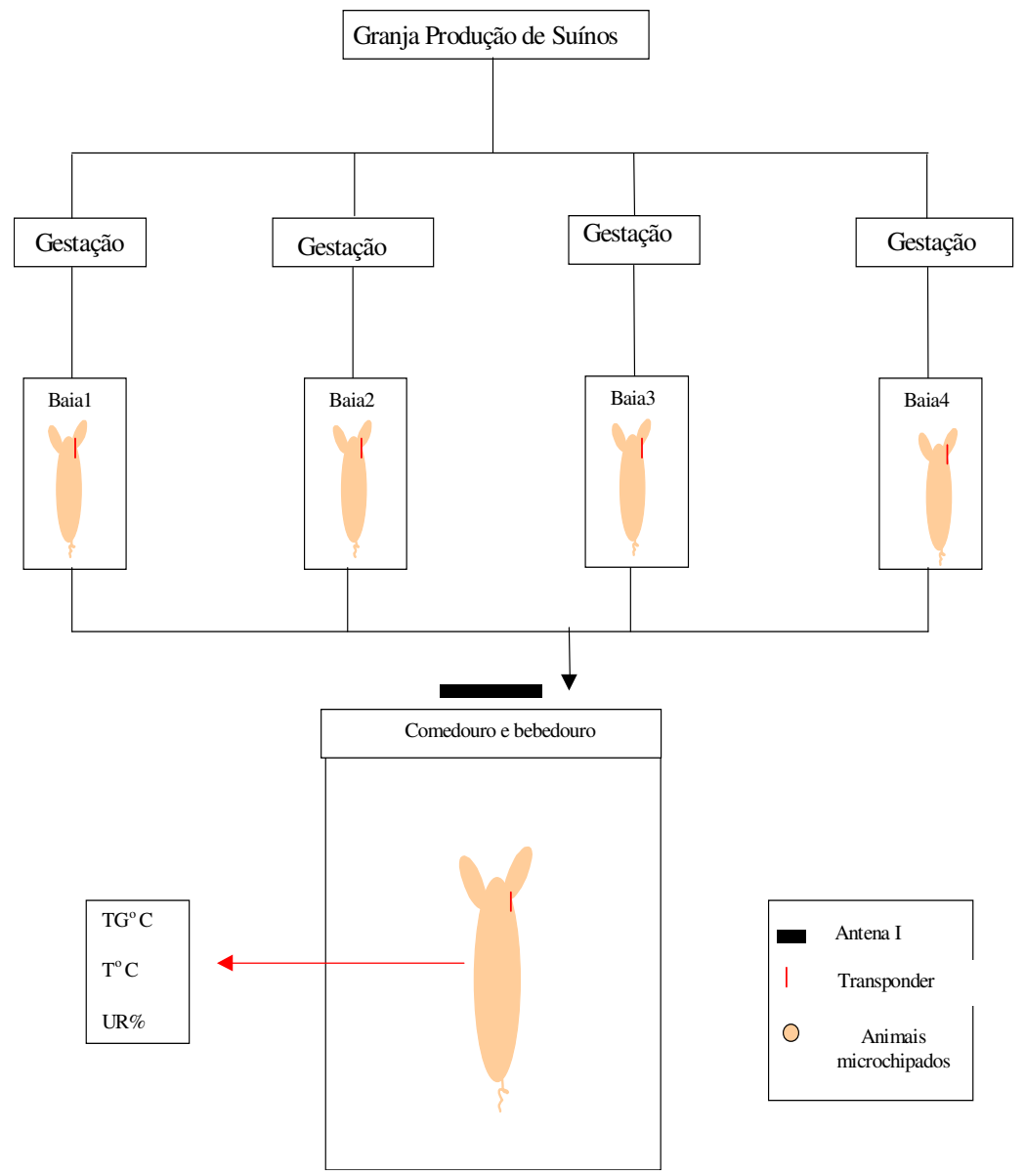

FIGURA 2 . Esquema geral da montagem do experimento, visando à interação comportamento versus ambiente.

Os dados foram coletados diariamente durante o período de permanência da matriz na gestação, mediante o armazenamento pelos equipamentos, transferidos para o computador via radiofreqüência, mediante o mesmo programa de gerenciamento, e os dados obtidos foram manipulados.

Com o registro dos dados por intermédio do desenvolvimento de um programa, pôde ser equacionada a frequiência de ida ao bebedouro, em função da variação das condições climáticas, de acordo com a seguinte função:

$$
\mathrm{P}=\mathrm{f}(\mathrm{TG}, \mathrm{Tbs}, \mathrm{Tbu}, \mathrm{UR}, \mathrm{X})
$$

em que,

$\mathrm{P}$ - freqüência de acesso ao comedouro ou bebedouro;

TG - temperatura de globo negro, ${ }^{\circ} \mathrm{C}$;

Tbs - temperatura de bulbo seco, ${ }^{\circ} \mathrm{C}$;

Tbu - temperatura de bulbo úmido, ${ }^{\circ} \mathrm{C}$;

UR - umidade relativa do ar, \%, e

$\mathrm{X}$ - variável dependente do sistema de produção. 
Para o desenvolvimento dessa função, foi utilizado o método estatístico modelo de regressão saturado, que utilizou dados referentes ao consumo de água e alimentos pelo animal de acordo com a temperatura e umidade do ar. Na granja onde foi realizada a pesquisa, os animais foram alimentados somente uma vez por dia, em horário rigidamente controlado, consumindo o alimento imediatamente após serem colocados. Como a matriz, na fase de gestação em gaiola, se alimenta todos os dias no mesmo horário, a freqüência de ida ao comedouro é $100 \%$ no horário em que é fornecida a alimentação, período que se inicia às $7 \mathrm{~h}$ da manhã e finaliza às $8 \mathrm{~h}$ da manhã. Sendo assim, o modelo desenvolvido representa somente a freqüência de ida ao bebedouro de acordo com as variáveis climáticas. Dessa maneira, os fatores ambientais devem ter efeitos visíveis somente por meio do consumo de água pelo animal.

Portanto, o desenvolvimento do programa computacional foi importante para receber o sinal transmitido pela porca, por meio do transponder, sendo esse enviado para as antenas que, em seguida, transmitia os dados para o software desenvolvido. Foram registrados, a cada medição, o número de identificação do animal, a data e a hora, e ainda a temperatura (TG, Tbs e Tbu) do local, registrada pelo datalogger.

\section{RESULTADOS E DISCUSSÃO}

Utilizou-se, para desenvolver o modelo, que forneceu o comportamento da porca de acordo com as variáveis climáticas, do método estatístico do modelo de regressão saturado.

O modelo forneceu a freqüência de ida ao bebedouro nas diferentes fases do dia e também a freqüência média ocorrida na zona de termoneutralidade da porca nesta fase [eq.(2)].

$$
\mathrm{C}=932-1,44 \mathrm{Tbs} \text { TG }-1,02 \mathrm{TG} \text { UR +0,0427 Tbs TG UR }
$$

em que,

C - freqüência média de ida ao bebedouro;

Tbs - temperatura de bulbo seco, ${ }^{\circ} \mathrm{C}$;

UR - umidade relativa, \%, e

$\mathrm{TG}$ - temperatura de globo negro, ${ }^{\circ} \mathrm{C}$.

Na Tabela 1, mostra-se que cada parâmetro é altamente significativo (inclusive para nível de significância de 0,01). A análise apresentou desvio-padrão para o modelo de 23,97 e coeficiente de ajuste (r-sq) de $81,8 \%$, que mede o quão bem ajustado está o modelo, sendo que o valor máximo é de 100\%. Foi feita a análise de variância, obtendo-se os dados da Tabela 2.

TABELA 1. Parâmetros fornecidos pelo teste de regressão, utilizado no modelo.

\begin{tabular}{ccccc}
\hline Predictor & Coeficiente & SE Coeficiente & T & P-valor \\
\hline Constante & 931,9 & 105,9 & 8,80 & 0,000 \\
tbs tg & $-1,4366$ & 0,1930 & $-7,44$ & 0,000 \\
$\operatorname{tg}$ ur & $-1,0168$ & 0,1356 & $-7,50$ & 0,000 \\
$\operatorname{tbs}$ tg u & 0,042688 & 0,005642 & 7,57 & 0,000 \\
\hline
\end{tabular}

TABELA 2. Resultados da análise de variância.

\begin{tabular}{crrrrc}
\hline Fonte & DF & SS & MS & F & P-valor \\
\hline Regressão & 3 & 134.694 & 44.898 & 19,53 & 0,000 \\
Erro residual & 13 & 29.884 & 2.299 & & \\
Total & 16 & 164.578 & & & \\
\hline
\end{tabular}


O p-valor desse teste mostra que o modelo tem, pelo menos, um parâmetro significativo, conforme visto anteriormente. O modelo é válido para porcas em gestação em gaiolas, usando-se as variáveis climáticas da região, para a freqüência de ida ao bebedouro.

As pressuposições do modelo estatístico de regressão foram verificadas por meio das análises de resíduos: homocedasticidades a variância (variância constante - estabilizada), normalidade e independência dos resíduos. Foi feito um teste de validação do modelo em que foram inseridos os dados médios de TG, UR e Tbs do período do estudo, no qual pôde ser observado o comportamento da porca dentro e fora da zona de termoneutralidade.

Observando-se as médias diárias dos dados coletados no experimento, verificou-se que somente dois dias apresentaram valores de temperatura e umidade dentro da zona de termoneutralidade. $\mathrm{O}$ restante dos dias ficou na zona crítica, considerando que a zona de termoneutralidade ótima para a porca está entre $12{ }^{\circ} \mathrm{C}$ e $20{ }^{\circ} \mathrm{C}$, com umidades entre $50 \%$ e $70 \%$, de acordo com ESMAY (1982), HAHN et al. (1987), POMAR et al. (1991) e NIENABER et al. (1987).

No gráfico Boxplot (Figura 3), obtido pelo software estatístico "Minitab", apresenta-se a preferência térmica das porcas dentro e fora da zona de termoneutralidade.

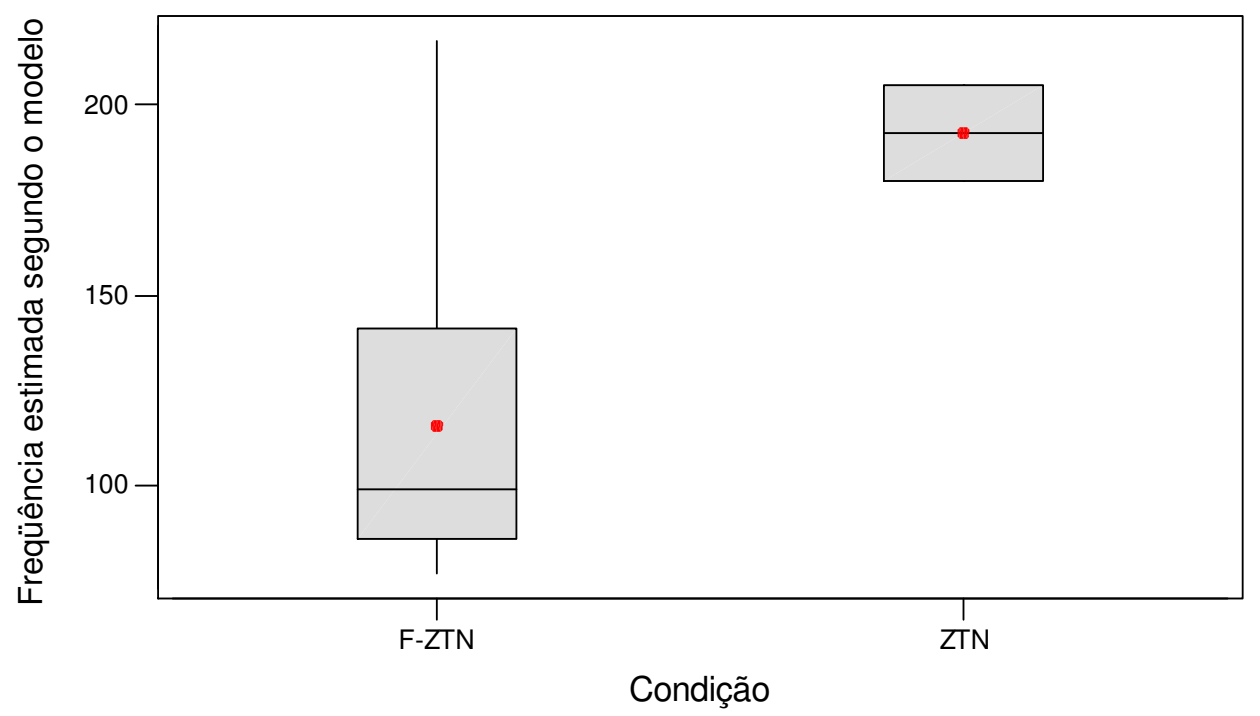

FIGURA 3. Padrão comportamental pela freqüência estimada do modelo para as condições ambientais: na Zona de Termoneutralidade (ZTN) e fora desta (F-ZTN).

Observa-se, na Figura 3, que a "caixa" que representa os dois valores da zona de termoneutralidade (ZTN) está bem acima da outra "caixa", que representa os dados fora da zona de termoneutralidade (F-ZTN). Como a identificação eletrônica somente identifica a posição em que se encontra o animal e não identifica qual a atividade que está sendo exercida por ele, neste trabalho, observou-se que a freqüência de ida ao bebedouro na zona de termoneutralidade (ZTN) foi maior quando comparado com os dados fora da zona de termoneutralidade (F-ZTN), não podendo informar se o animal estava consumindo água ou se estava apenas posicionado perto do bebedouro.

Por meio desse modelo, foram obtidas as frequiências médias diárias de ida ao bebedouro, de acordo com as variáveis climáticas, dispondo-se da identificação eletrônica como ferramenta indispensável de acordo com PANDORFI (2002). 


\section{CONCLUSÕES}

O modelo matemático desenvolvido permitiu avaliar com precisão e sem interferência humana a interação entre a preferência térmica de matrizes de suínos, em função dos dados climáticos do ambiente, utilizando como ferramenta a identificação eletrônica.

\section{AGRADECIMENTOS}

À Fundação de Amparo à Pesquisa do Estado de São Paulo (FAPESP), pelo apoio financeiro ao trabalho, e à Granja Querência, pela oportunidade do desenvolvimento da pesquisa.

\section{REFERÊNCIAS}

BELLAVER, C. Segurança alimentar e controle de qualidade no uso de ingredientes para alimentação dos suínos. Conferência Internacional Virtual sobre Qualidade de Carne Suína. Disponível em: <http://www.conferencia.uncnet.br/pork/seg/pal/anais01p2_bellaver_pt.pdf>. Acesso em: $1^{\circ}$ dez. 2001.

ESMAY, M.L. Principles of animal environment. Westport: Avi Publishing Company, 1982. 325 p.

HAHN, G.L.; NIENABER, J.A.; DESHAZER, J.A. Air temperature influences on swine performance and behavior. Applied Engineerning in Agriculture ASAE, St.Joseph, v.3, n.2, p.295-302, 1987.

HAMRITA, T. K.; WICKLEN, G. Van; CZARICK, M.; LACY, M. Monitoring poultry deep body temperature using biotelemetry. Applied Engineering in Agriculture ASAE, St. Joseph, v.14, n.3, p.327-31, 1998.

KORTHALS, R.L.; McDONALD, T.P.; EIGENBERG, R.A. Experiences with transponders for monitoring bioenergetic responses. In: ASAE MEETING PRESENTATION, 1992, Charlotte. Anais...Charlotte: ASAE, 1992. 1 CD-ROM.

LEITE, D.M.G. Software para gerenciamento de granjas suinícolas. In: CONGRESSO E MOSTRA DE AGROINFORMÁTICA - INFOAGRO, 1., 2000, Ponta Grossa. Anais...Ponta Grossa: Universidade Estadual de Ponta Grossa, 2000. 1 CD-ROM.

NÄÄS, I.A.; CURTO, F.P.F.; FIRME, F.P.; PEREIRA, D.F.; AMENDOLA, M.; BEHRENS, F.H. Using transponders for determining breeder behavior versus environmental temperature. In: INTERNATIONAL SYMPOSIUM ON LIVESTOCK ENVIRONMENT, 6., 2001, Louisville. Proceedings... St Joseph: Society for Engineering in Agricultural, Food and Biological Systems, 2001a. v.1, p.626-31.

NÄÄS, I.A.; PEREIRA, D.F.; CURTO, F.P.F.; BEHRENS, F.H.; AMENDOLA, M.; MURAYAMA, M.C.; MANTOVANI, E.C. The using of EID for understanding female broiler breeders behavior in two different housing solar orientation. In: THE WORLD CONFERENCE ON COMPUTER IN AGRICULTURE AND NATURAL RESOURCES, 1., 2001, Foz do Iguaçu. Proceedings... St. Joseph: Society for Engineering in Agricultural, Food and Biological Systems, 2001b. 1 CD-ROM.

NIENABER, J.A.; HAHN, L.G.; YEN, J.T. Thermal environment effects on growing-finishing swine. Part I - Growth, feed intake and heat production. Transactions of the ASAE, St. Joseph, v.30, n.6, p.1772-5, 1987.

PANDORFI, H. Avaliação do comportamento de leitões em diferentes sistemas de aquecimento por meio da análise de imagens e identificação eletrônica. 2002. 88 f. Dissertação (Mestrado em Física do Ambiente Agrícola) - Escola Superior de Agricultura “Luiz de Queiroz”, Universidade de São Paulo, Piracicaba, 2002. 
POMAR, C.; HARRIS, D.L.; SAVOIE, P.; MINVIELLE, F. Computer simulation model of swine production systems. Journal of Animal Science, Quebec, v.69, p.2822-36, 1991.

SILVA, K.O.; NÄÄS, I.A.; SALGADO, D.D. The ID microchip migration in piglets. In: CONGRESS OF THE INTERNATIONAL PIG VETERINARY SOCIETY, 17., 2002, Iowa. Proceedings... Iowa: ISU, 2002. p.439. 